\title{
A Predictive Location Tracking Algorithm for Mobile Devices with Deficient Signal Sources
}

\author{
Yu-Chiun Lin, Po-Hsuan Tseng, and Kai-Ten Feng \\ Department of Communication Engineering \\ National Chiao Tung University \\ Hsinchu, Taiwan \\ dadun.cm90@nctu.edu.tw, walker.cm90@nctu.edu.tw, and ktfeng@mail.nctu.edu.tw
}

\begin{abstract}
Location estimation and tracking for the mobile devices have attracted a significant amount of attention in recent years. The network-based location estimation schemes have been widely adopted based on the radio signals between the mobile device and the base stations. The location estimators associated with the Kalman filtering techniques are exploited to both acquire location estimation and trajectory tracking for the mobile devices. However, most of the existing schemes become unapplicable due to the insufficiency of signal sources. In this paper, a Predictive Location Tracking (PLT) algorithm is proposed to alleviate this problem. The predictive information obtained from the Kalman filter is employed to provide the additional signal inputs for the location estimators. The proposed PLT scheme can offer persistent accuracy for location tracking of the mobile devices, especially with inadequate signal sources. Numerical results demonstrate that the proposed PLT algorithm can achieve better precision, comparing with other existing schemes, in mobile location estimation and tracking.
\end{abstract}

\section{INTRODUCTION}

Wireless location technologies, which are designated to estimate the position of a Mobile Station (MS), have drawn a lot of attention over the past few decades. Different types of Location-Based Services (LBSs) have been proposed and studied, including the emergency 911 (E-911) subscriber safety services [1], the location-based billing, the navigation system, and applications for the Intelligent Transportation System (ITS) [2]. Due to the emergent interests in the LBSs, it is required to provide enhanced precision in the location estimation of a MS under different environments.

A variety of wireless location techniques have been investigated [3]. The network-based location estimation schemes have been widely proposed and employed in the wireless communication system. These schemes locate the position of a MS based on the measured radio signals from its neighborhood Base Stations (BSs). The representative algorithms for the network-based location estimation techniques are the Time-OfArrival (TOA), the Time Difference-Of-Arrival (TDOA), and the Angle-Of-Arrival (AOA). The TOA scheme measures the arrival time of the radio signals coming from different wireless BSs; while the TDOA scheme measures the time difference between the radio signals. The AOA technique is conducted

\footnotetext{
${ }^{1}$ This work was in part funded by the MOE ATU Program 95W803C, NSC 95-2218-E-009-014, and the MediaTek research center at the National Chiao Tung University.
}

within the BS by observing the arriving angle of the signals coming from the MS.

In addition to the estimation of a MS's position, trajectory tracking of a moving MS has been studied [4] - [8]. The technique by combining the Kalman filter with the Weighted Least Square (WLS) method is exploited in [4]. The Kalman Tracking (KT) scheme [5] [6] distinguishes the linear part from the originally nonlinear equations for location estimation. The linear aspect is exploited within the Kalman filtering formulation; while the nonlinear term is served as an external measurement input to the Kalman filter. The technique utilized in [7] adopted the Kalman filters for both pre-processing and post-processing in order to both mitigate the NLOS noises and track the MS's trajectory. The Cascade Location Tracking (CLT) scheme as proposed in [8] utilizes the two-step Least Square (LS) method [9] [10] for initial location estimation of the MS. The Kalman filtering technique is employed to smooth out and to trace the position of the MS based on its previously estimated data.

However, the wireless location tracking problem with insufficient signal sources has not been addressed in previous studies. In the cellular-based networks, three BSs are required in order to provide three signal sources for the TOA-based location estimation. Nevertheless, the scenario with sufficient signal sources does not always happen in real circumstances, e.g. under rural environments or city valley with blocking buildings. It will be beneficial to provide consistent accuracy for location tracking under various environments. In this paper, a Predictive Location Tracking (PLT) algorithm is proposed to improve the problem with insufficient measurement inputs. The predictive information obtained from the Kalman filter is adopted as the virtual signal sources, which are incorporated into the two-step LS method for location estimation and tracking. Consistent precision for location tracking of a MS is observed by exploiting the proposed PLT algorithm.

The remainder of this paper is organized as follows. Section II describes the modeling of the signal sources and the twostep LS estimator. The proposed Predictive Location Tracking (PLT) algorithm is explained in Section III. Section IV illustrates the performance evaluation of the proposed PLT scheme in comparison with the KT and the CLT techniques. Section $\mathrm{V}$ draws the conclusions. 


\section{PRELIMINARIES}

\section{A. Mathematical Modeling}

In order to facilitate the design of the proposed PLT algorithm, the signal model for the TOA measurements is utilized. The set $\boldsymbol{r}_{k}$ contains all the available measured relative distance at the $k^{t h}$ time step, i.e. $\boldsymbol{r}_{k}=\left\{r_{1, k}, \ldots, r_{i, k}, \ldots, r_{N_{k}, k}\right\}$, where $N_{k}$ denotes the number of available BSs at the time step $k$. The measured relative distance $\left(r_{i, k}\right)$ between the MS and the $i^{t h} \mathrm{BS}$ (obtained at the $k^{t h}$ time step) can be represented as

$$
r_{i, k}=c \cdot t_{i, k}=\zeta_{i, k}+n_{i, k}+e_{i, k} \quad i=1,2, \ldots, N_{k}
$$

where $t_{i, k}$ denotes the TOA measurement obtained from the $i^{\text {th }} \mathrm{BS}$ at the $k^{\text {th }}$ time step, and $c$ is the speed of light. $r_{i, k}$ is contaminated with the TOA measurement noise $n_{i, k}$ and the Non-line-of-sight (NLOS) error $e_{i, k}$. The noiseless relative distance $\zeta_{i, k}$ between the MS and the $i^{t h}$ BS can be obtained as

$$
\zeta_{i, k}=\left[\left(x_{k}-x_{i, k}\right)^{2}+\left(y_{k}-y_{i, k}\right)^{2}\right]^{\frac{1}{2}}
$$

where $\boldsymbol{x}_{k}=\left[\begin{array}{ll}x_{k} & y_{k}\end{array}\right]$ represents the MS's position and $\boldsymbol{x}_{i, k}=$ $\left[\begin{array}{ll}x_{i, k} & y_{i, k}\end{array}\right]$ is the location of the $i^{t h} \mathrm{BS}$.

\section{B. The Two-Step LS Estimator}

The two-step LS scheme is utilized as the location estimator for the proposed PLT algorithm. It is noticed that three TOA measurements are required for the two-step LS method in order to solve for the location estimation problem. The concept of the two-step LS method is to acquire an intermediate location estimate in the first step with the definition of a new variable $\beta_{k}$, which is mathematically related to the MS's position, i.e. $\beta_{k}=x_{k}^{2}+y_{k}^{2}$. At this stage, the variable $\beta_{k}$ is assumed to be uncorrelated to the MS's position. This assumption effectively transforms the nonlinear equations for location estimation into a set of linear equations, which can be directly solved by the LS method.

The second step of the method primarily considers the relationship that the variable $\beta_{k}$ is equal to $x_{k}^{2}+y_{k}^{2}$, which was originally assumed to be uncorrelated in the first step. An improved location estimation can be obtained after the adjustment from the second step. The detail algorithm of the two-step LS method for location estimation can be found in [10].

\section{The Proposed Predictive Location Tracking (PLT) AlgORITHM}

In this section, the proposed PLT scheme is described. The concepts and motivations of the proposed scheme is explained in Subsection $A$. Subsection $B$ summarizes the formulation of the PLT algorithm with two different scenarios.

\section{A. Overview of the PLT Algorithm}

The objective of the proposed PLT algorithm is to utilize the predictive information from the Kalman filter to serve as the assisted measurement inputs under the environments with deficient signal sources. Fig. 1 illustrates the system architectures of the KT [5], the CLT [8] and the proposed PLT schemes. The TOA signals are acquired as the signal inputs to each of the system, which results in the estimated state vector of the MS, i.e. $\hat{\boldsymbol{s}}_{k}=\left[\begin{array}{lll}\hat{\boldsymbol{x}}_{k} & \hat{\boldsymbol{v}}_{k} & \hat{\boldsymbol{a}}_{k}\end{array}\right]^{T}$ where $\hat{\boldsymbol{x}}_{k}=\left[\begin{array}{ll}\hat{x}_{k} & \hat{y}_{k}\end{array}\right]$ represents the MS's estimated position, $\hat{\boldsymbol{v}}_{k}=\left[\hat{v}_{x, k} \hat{v}_{y, k}\right]$ is the estimated velocity, and $\hat{\boldsymbol{a}}_{k}=\left[\hat{a}_{x, k} \hat{a}_{y, k}\right]$ denotes the estimated acceleration.

Since the equations associated with the network-based location estimation are inherently nonlinear, different mechanisms are considered within the existing algorithms for location tracking. The KT scheme [5] (as shown in Fig. 1.(a)) explores the linear aspect of location estimation within the Kalman filtering formulation. The nonlinear term (i.e. $\beta_{k}=x_{k}^{2}+y_{k}^{2}$ ) is treated as a measurement input to the Kalman filter by obtaining from an external location estimator, e.g. the twostep LS method. On the other hand, the CLT scheme [8] (as in Fig. 1.(b)) adopts the two-step LS method to acquire the preliminary location estimate of the MS. The Kalman Filter is utilized to smooth out the estimation error by tracking the state vector $\hat{\boldsymbol{s}}_{k}$ of the MS.

The architecture of the proposed PLT scheme is illustrated in Fig. 1.(c). It can be seen that the PLT algorithm becomes the CLT scheme as $N_{k} \geq 3$. On the other hand, the effectiveness of the PLT scheme is revealed while $1 \leq N_{k}<3$. The predictive state information obtained from the Kalman filter is fed back into the two-step LS estimator. The extended set for the measured relative distance becomes $\boldsymbol{r}_{k}^{e}=\left\{\boldsymbol{r}_{k}, \boldsymbol{r}_{v, k}\right\}$, with

$$
\boldsymbol{r}_{v, k}= \begin{cases}\left\{r_{v_{1}, k}\right\} & \text { for } N_{k}=2 \\ \left\{r_{v_{1}, k}, r_{v_{2}, k}\right\} & \text { for } N_{k}=1\end{cases}
$$

where $\boldsymbol{r}_{v, k}$ is defined as the set of the virtual measurement inputs obtained from the prediction mechanism of the Kalman filter.

In both the KT and the CLT schemes, the state vector $\hat{\boldsymbol{s}}_{k}$ can only be updated by the internal prediction mechanism of the Kalman filter while $N_{k}<3$ (as shown in Fig. 1 with the dashed lines). The location estimator (i.e. the two-step LS method) is disabled owing to the inadequate number of the signal sources. The tracking capabilities of both schemes significantly depend on the correctness of the Kalman filter's prediction mechanism. Therefore, the performance for location tracking is severely degraded due to the changing behavior of the MS, i.e. with variations of the MS's acceleration. On the other hand, the proposed PLT algorithm can still provide satisfactory tracking performance with deficient measurement inputs, i.e. with $N_{k}=1$ and 2. Under these circumstances, the location estimator is still effective with the additional virtual measurements $\boldsymbol{r}_{v_{i}, k}$, which are imposed from the output of the Kalman filter (as shown in Fig. 1.(c)). It is noted that the PLT scheme performs the same as the CLT method under the case 


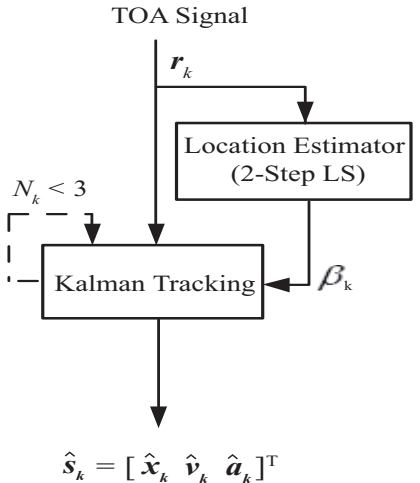

(a)

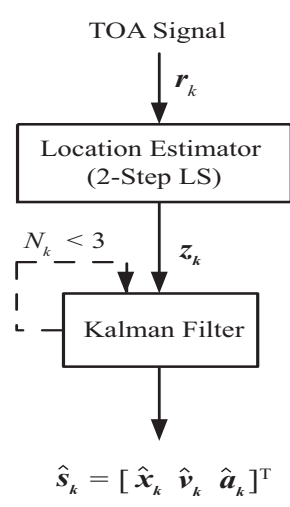

(b)

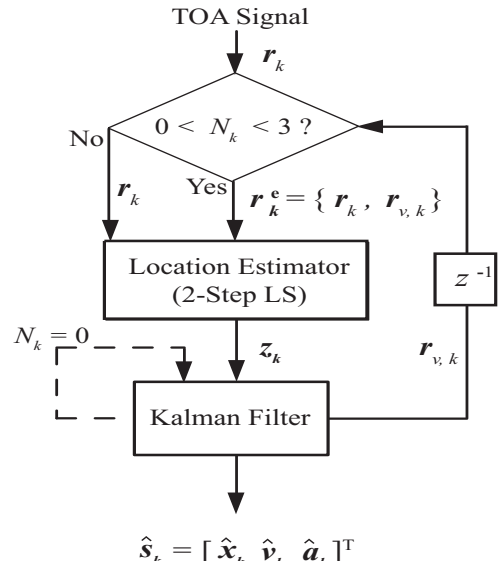

(c)

Fig. 1. The Architecture Diagrams of (a) the Kalman Tracking (KT) Scheme; (b) the Cascade Location Tracking (CLT) Scheme; and (c) the Proposed Predictive Location Tracking (PLT) Scheme

of no signal input, i.e. $N_{k}=0$. The computation of the values within the set $\boldsymbol{r}_{v, k}$ for the two different cases (as in (3)) will be presented in the next subsection.

\section{B. Formulation of the PLT Algorithm}

As shown in Fig. 1.(c), the measurement and state equations for the Kalman filter can be represented as

$$
\begin{aligned}
& \boldsymbol{z}_{k}=\mathbf{M} \hat{\boldsymbol{s}}_{k}+\boldsymbol{m}_{k} \\
& \hat{\boldsymbol{s}}_{k}=\mathbf{F} \hat{\boldsymbol{s}}_{k-1}+\boldsymbol{p}_{k}
\end{aligned}
$$

where $\hat{\boldsymbol{s}}_{k}=\left[\begin{array}{lll}\hat{\boldsymbol{x}}_{k} & \hat{\boldsymbol{v}}_{k} & \hat{\boldsymbol{a}}_{k}\end{array}\right]^{T}$. The variables $\boldsymbol{m}_{k}$ and $\boldsymbol{p}_{k}$ denote the measurement and the process noises associated with the covariance matrices $\mathbf{R}$ and $\mathbf{Q}$ within the Kalman filtering formulation. The measurement vector $\boldsymbol{z}_{k}=\left[\begin{array}{ll}\hat{x}_{l s, k} & \hat{y}_{l s, k}\end{array}\right]^{T}$ represents the measurement input obtained from the output of the two-step LS estimator at the $k^{t h}$ time step. The matrix $\mathbf{M}$ and the state transition matrix $\mathbf{F}$ can be obtained as

$$
\begin{aligned}
\mathbf{M}= & {\left[\begin{array}{cccccc}
1 & 0 & 0 & 0 & 0 & 0 \\
0 & 1 & 0 & 0 & 0 & 0
\end{array}\right] } \\
\mathbf{F}= & {\left[\begin{array}{cccccc}
1 & 0 & \Delta t & 0 & \frac{1}{2} \Delta t^{2} & 0 \\
0 & 1 & 0 & \Delta t & 0 & \frac{1}{2} \Delta t^{2} \\
0 & 0 & 1 & 0 & \Delta t & 0 \\
0 & 0 & 0 & 1 & 0 & \Delta t \\
0 & 0 & 0 & 0 & 1 & 0 \\
0 & 0 & 0 & 0 & 0 & 1
\end{array}\right] }
\end{aligned}
$$

where $\Delta t$ denotes the sample time interval. The main concept of the proposed PLT scheme is to provide additional virtual measurements (i.e. $\boldsymbol{r}_{v, k}$ as in (3)) to the two-step LS estimator while the signal sources are insufficient. Two cases (i.e. the two-BSs case and the single-BS case) are considered as follows:

1) The Two-BSs Case: As shown in Fig. 2, it is assumed that only two BSs associated with two TOA measurements are available at the time step $k$ in consideration. The main target is to introduce an additional virtual measurement, i.e. $\boldsymbol{r}_{v, k}=\left\{r_{v_{1}, k}\right\}$, which can be acquired from the output of the

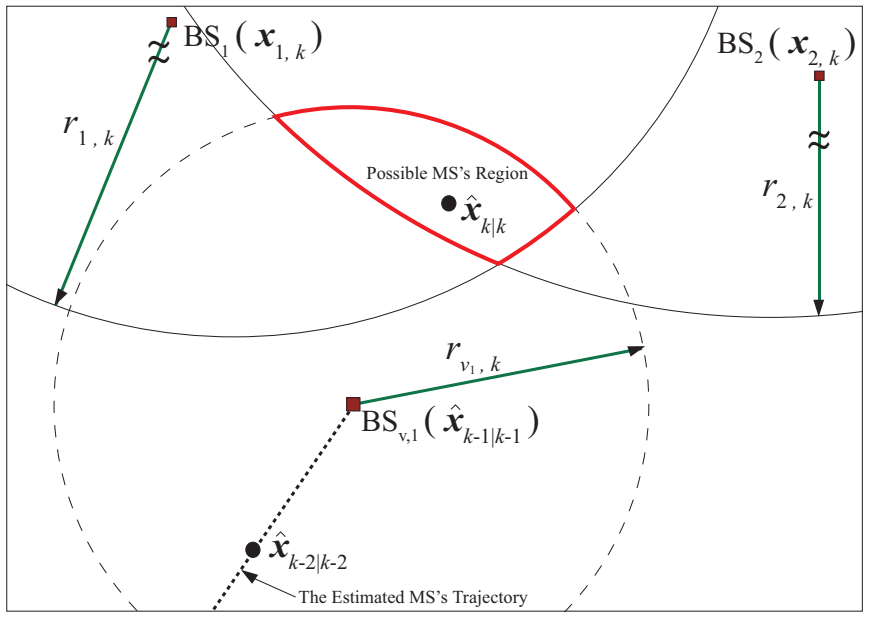

Fig. 2. The Schematic Diagram of the Two-BSs Case

Kalman filter. Knowing that there are predicting and correcting phases within the Kalman filtering formulation, the predictive state can therefore be utilized to compute the supplementary virtual measurement $r_{v_{1}, k}$ as

$$
\begin{aligned}
r_{v_{1}, k} & =\left\|\hat{\boldsymbol{x}}_{k \mid k-1}-\hat{\boldsymbol{x}}_{k-1 \mid k-1}\right\| \\
& =\left\|\mathbf{M F} \hat{\boldsymbol{s}}_{k-1 \mid k-1}-\hat{\boldsymbol{x}}_{k-1 \mid k-1}\right\|
\end{aligned}
$$

where $\hat{\boldsymbol{x}}_{k \mid k-1}$ denotes the predicted MS's position at the $k^{t h}$ step; while $\hat{\boldsymbol{x}}_{k-1 \mid k-1}$ is the corrected MS's position at the $(k-1)^{t h}$ time step. The virtual measurement $r_{v_{1}, k}$ is defined by taking the previous location estimate $\left(\hat{\boldsymbol{x}}_{k-1 \mid k-1}\right)$ as the position of the virtual BS (i.e. $\mathrm{BS}_{v, 1}$ ) with the predicted MS's position $\left(\hat{\boldsymbol{x}}_{k \mid k-1}\right)$ as the virtual position of the MS (as shown in Fig. 2). By adopting $r_{v_{1}, k}$ (in (8)) as the additional measurement input, the state vector $\hat{\boldsymbol{s}}_{k \mid k}$ can be acquired after the three measurement inputs $\boldsymbol{r}_{k}^{e}=\left\{r_{1, k}, r_{2, k}, r_{v_{1}, k}\right\}$ have been imposed into the two-step LS estimator, along with the implementation of the correcting phase of the Kalman filter at 


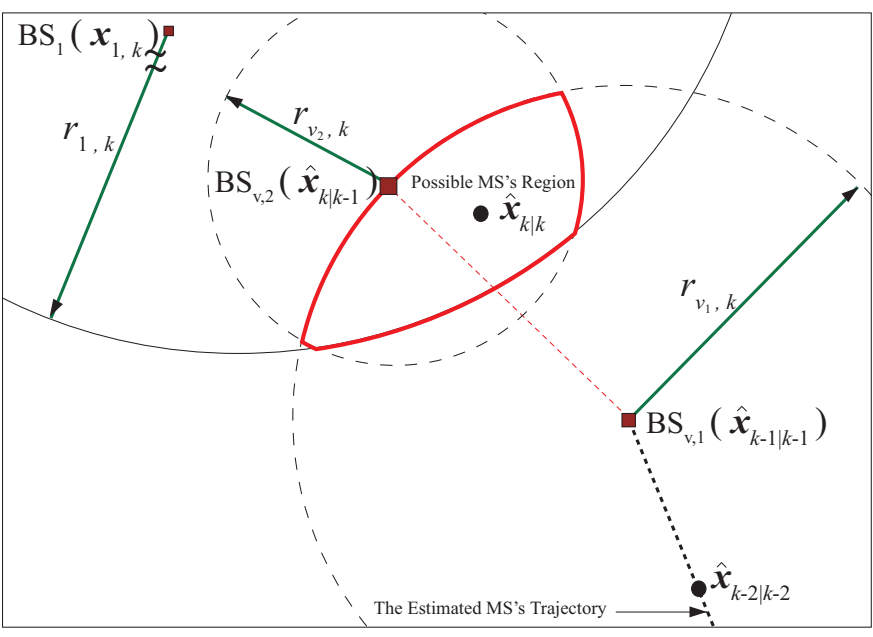

Fig. 3. The Schematic Diagram of the Single-BS Case

the time step $k$ as

$$
\begin{aligned}
\hat{\boldsymbol{s}}_{k \mid k}= & \hat{\boldsymbol{s}}_{k \mid k-1}+ \\
& \mathbf{P}_{k \mid k-1} \mathbf{M}^{T}\left[\mathbf{M} \mathbf{P}_{k \mid k-1} \mathbf{M}^{T}+\mathbf{R}\right]^{-1}\left(z_{k}-\mathbf{M} \hat{\boldsymbol{s}}_{k \mid k-1}\right)
\end{aligned}
$$

where

$$
\begin{aligned}
\mathbf{P}_{k \mid k-1}= & \mathbf{F} \mathbf{P}_{k-1 \mid k-1} \mathbf{F}^{T}+\mathbf{Q} \\
\mathbf{P}_{k-1 \mid k-1}= & {\left[\mathbf{I}-\mathbf{P}_{k-1 \mid k-2} \mathbf{M}^{T}\right.} \\
& \left.\left(\mathbf{M P}_{k-1 \mid k-2} \mathbf{M}^{T}+\mathbf{R}\right)^{-1} \mathbf{M}\right] \mathbf{P}_{k-1 \mid k-2}
\end{aligned}
$$

It is noted that $\mathbf{P}_{k \mid k-1}$ and $\mathbf{P}_{k-1 \mid k-1}$ represent the predicted and the corrected estimation covariances within the Kalman filter. As can been observed from Fig. 2, $r_{v_{1}, k}$, associated with $r_{1, k}$ and $r_{2, k}$, provides a confined region for the estimation of the MS's location at the time step $k$.

2) The Single-BS Case: In this case, only one BS with one TOA measurement input is available at the $k^{t h}$ time step (as shown in Fig.3). Two additional virtual measurements are required for the computation of the two-step LS estimator, i.e. $\boldsymbol{r}_{v, k}=\left\{r_{v_{1}, k}, r_{v_{2}, k}\right\}$. Similar to the previous case, the first virtual measurement $r_{v_{1}, k}$ is acquired by utilizing $\hat{\boldsymbol{x}}_{k-1 \mid k-1}$ as the position of $\mathrm{BS}_{v, 1}$ with the virtual MS's position as $\hat{\boldsymbol{x}}_{k \mid k-1}$ (as in (8)). The second virtual measurement $r_{v_{2}, k}$ is defined as the average prediction error from the Kalman filtering formulation from the previous time steps. As illustrated in Fig. 3 , the second virtual BS's position (i.e. $\mathrm{BS}_{v, 2}$ ) is assumed to locate at the predicted MS's position $\hat{\boldsymbol{x}}_{k \mid k-1}$ with the virtual measurement $r_{v_{2}, k}$ obtained as

$$
r_{v_{2}, k}=\frac{1}{k-1} \sum_{i=1}^{k-1}\left\|\hat{\boldsymbol{x}}_{i \mid i}-\hat{\boldsymbol{x}}_{i \mid i-1}\right\|
$$

It is noted that $r_{v_{2}, k}$ is obtained as the mean prediction error until the $(k-1)^{t h}$ time step. In the case while the Kalman filter provides sufficient accuracy in its prediction phase, the value of $r_{v_{2}, k}$ may approach zero. Associated with the single measurement $r_{1, k}$ from $\mathrm{BS}_{1}$ (as in Fig. 3), the two additional virtual measurements $r_{v_{1}, k}$ (centered at $\hat{\boldsymbol{x}}_{k-1 \mid k-1}$ ) and $r_{v_{2}, k}$ (centered at $\hat{\boldsymbol{x}}_{k \mid k-1}$ ) provide the restricted region for the location estimation of the MS under insufficient signal sources.

It is noticed that the variations of the measurement inputs are the required information for adopting the two-step LS estimator. It utilizes the signal variation as an indicator to consider the weighting for a specific signal source, i.e. in order to perform the weighted least square algorithm. Similar concept can be exploited to the virtual measurement cases. The signal variation of $r_{v_{1}, k}$ considers the variance of the predicted distance $\left\|\hat{\boldsymbol{x}}_{k \mid k-1}-\hat{\boldsymbol{x}}_{k-1 \mid k-1}\right\|$ from the previous $k-1$ time steps; while the variation of $r_{v_{2}, k}$ is denoted as the variance of the prediction errors.

\section{Performance Evaluation}

Simulations are performed to show the effectiveness of the proposed PLT scheme under different numbers of BSs, including the scenarios with deficient signal sources. The noise models and the simulation parameters are illustrated in Subsection $A$. The performance comparison between the proposed PLT algorithm with the other existing location tracking schemes, i.e. the Kalman Tracking (KT) and the Cascade Location Tracking (CLT) schemes, are conducted in Subsection $B$.

\section{A. The Noise Models and the Simulation Parameters}

Different noise models [11] [12] for the the TOA measurements are considered in the simulations. The model for the measurement noise of the TOA signals is selected as the Gaussian distribution with zero mean and 60 meters of standard deviation, i.e. $n_{i, k} \sim \mathcal{N}(0,3600)$. On the other hand, an exponential distribution $p_{e_{i, k}}(\tau)$ is assumed for the NLOS noise model of the TOA measurements as

$$
p_{n_{i, k}}(v)= \begin{cases}\frac{1}{\lambda_{i}} \exp \left(-\frac{v}{\lambda_{i}}\right) & v>0 \\ 0 & \text { otherwise }\end{cases}
$$

where $\lambda_{i}=c \cdot \tau_{i}=c \cdot \tau_{m} \zeta_{i}^{\varepsilon} \rho$. The parameter $\tau_{i}$ is the RMS delay spread between the $i^{\text {th }}$ BS to the MS, and $\tau_{m}$ is the median value of $\tau_{i}$, which is selected as 0.1 in the simulations. $\varepsilon$ is the path loss exponent which is assumed to be 0.5 , and the factor for shadow fading $\rho$ is set to 1 in the simulations. The parameters for the noise models as listed in this subsection primarily fulfill the environment while the MS is located within the rural area. It is noticed that the reason for selecting the rural area as the simulation scenario is due to its higher probability to suffer from deficiency of signal sources. Moreover, the sampling time $\Delta t$ is chosen as $1 \mathrm{sec}$ in the simulations.

\section{B. Simulation Results}

The performance comparison between the KT scheme, the CLT scheme, and the proposed PLT algorithm are conducted under the rural environment. Figs. 4 illustrates the performance comparison of the trajectory tracking using these three 


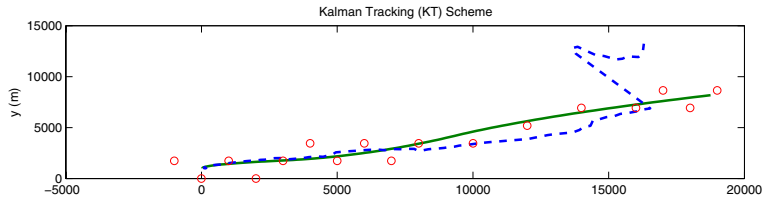

Cascaded Location Tracking (CLT) Scheme

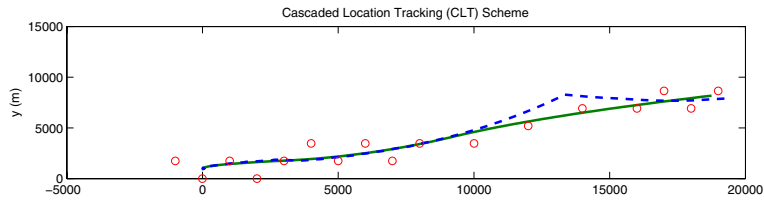

dictive Location Tracking (PLT) Sche

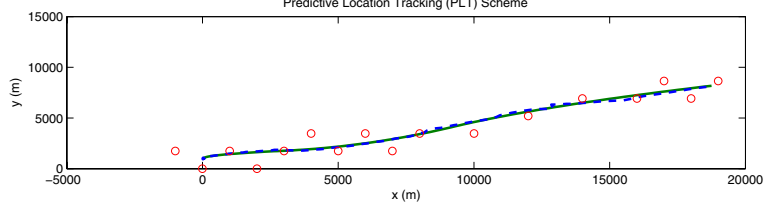

Fig. 4. Trajectory Tracking of the MS using the KT (Top Plot), the CLT (Middle Plot), and the PLT (Bottom Plot) Schemes (Solid Lines: True Trajectories; Dashed Lines: Estimated Trajectories; Red Empty Circles: the Position of the BSs)

schemes. It is noted that the number of BSs becomes insufficient (i.e. $N_{k}<3$ ) between the time interval of $t=72$ to 109 sec. The total simulation interval is set as 120 seconds. During the time interval between $t=72$ to $109 \mathrm{sec}$ with inadequate signal sources, only the proposed PLT scheme can achieve satisfactory performance in the trajectory tracking. Both the KT and the CLT schemes diverge from the true trajectory due to the inadequate number of measurement inputs, even though the number of BSs becomes sufficient afterwards (i.e. from $t=$ 110 to $120 \mathrm{sec}$ ). The effectiveness of the proposed PLT scheme is observed, especially under insufficient signal sources.

Fig. 5 shows the sorted location estimation errors (i.e. $\left.\Delta x_{k}=\left\|\hat{\boldsymbol{x}}_{k}-\boldsymbol{x}_{k}\right\|\right)$ obtained from the three schemes. Since the PLT algorithm is essentially the same as the CLT scheme while the number of BSs is adequate, both schemes perform the same under $60 \%$ of position errors. The performance of the CLT scheme becomes worse after $70 \%$ of position errors due to the deficiency of signal sources; while the proposed PLT algorithm can still provide feasible performance for location tracking. Moreover, the performance obtained from the KT scheme is comparably worse than the CLT and the PLT algorithms, i.e. around $200 \mathrm{~m}$ worse under $50 \%$ of position error. The main reason can be attributed to the difference between the inherent structures of the KT and the CLT schemes. The KT scheme is more focused on the compromise between the estimated states $\hat{\boldsymbol{s}}_{k}$ (i.e. between the position, the velocity, and the acceleration) with its measurement inputs $\boldsymbol{r}_{k}$; while the CLT scheme is targeting on smoothing the location trajectory of the MS. Therefore, the CLT algorithm can achieve better position estimation and tracking comparing with the KT scheme.

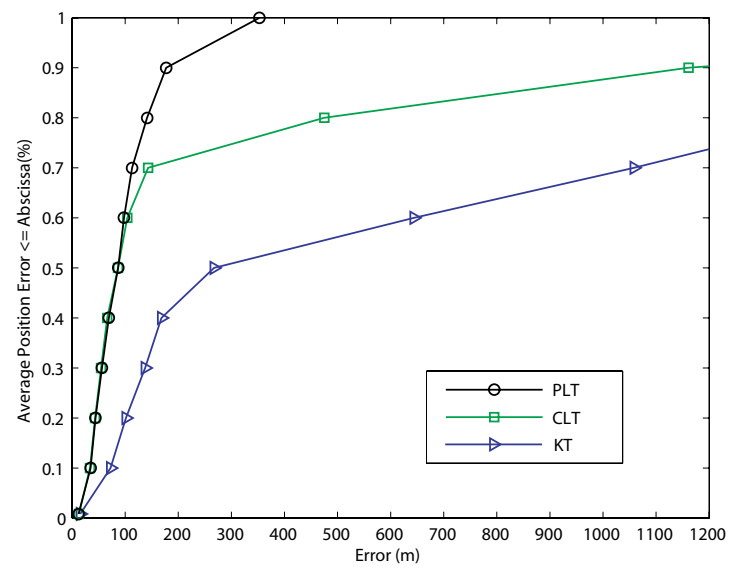

Fig. 5. Performance Comparison between the Location Tracking Schemes

\section{CONClusion}

In this paper, a Predictive Location Tracking (PLT) algorithm is proposed. The predictive information obtained from the Kalman filtering formulation is exploited as the additional measurement inputs for the location estimator. It is shown in the simulation results that the proposed PLT scheme can provide consistent accuracy for location estimation and tracking even with insufficient signal sources.

\section{REFERENCES}

[1] "Revision of the Commissions Rules to Insure Compatibility with Enhanced 911 Emergency Calling Systems," Federal Communications Commission, 1996.

[2] S. Feng and C. L. Law, "Assisted GPS and Its Impact on Navigation in Intelligent Transportation Systems," IEEE Intelligent Transportation Systems, 2002, pp. 926-931.

[3] Y. Zhao, "Standardization of Mobile Phone Positioning for 3G Systems," IEEE Communications Magazine, vol. 40, July 2002, pp. 108-116.

[4] J. Shuhong, S. Xicai, K. Fanru, "A Time-of Arrival Location Algorithm for Maneuvering Target on Two-dimensional Surface," IEEE International Conference on Signal Processing, vol. 2, October 1998, pp. 1700-1703.

[5] M. Nájar and J. Vidal, "Kalman Tracking Based on TDOA for UMTS mobile Location," IEEE International Symposium on Personal, Indoor and Mobile Radio Communications, vol. 1, September 2001, pp. B-4549.

[6] M. Nájar and J. Vidal, "Kalman Tracking for Mobile Location In NLOS Situations," IEEE International Symposium on Personal, Indoor and Mobile Radio Communications Proceedings, vol. 3, 7-10 Septemebr 2003, pp. 2203-2207.

[7] B. L. Le, K. Ahmed, and H. Tsuji, "Mobile Location Estimator with NLOS Mitigation Using Kalman Filtering," IEEE Wireless Communications and Networking, vol. 3, March 2003, pp. 1969-1973.

[8] C. L. Chen and K. F. Feng, "Hybrid location estimation and tracking system for mobile devices," IEEE Vehicular Technology Conference (VTC) Spring 2005, Vol. 4, June 2005, pp. 2648-2652.

[9] Y. T. Chan and K. C. Ho, "A Simple and Efficient Estimator for Hyperbolic Location," IEEE Trans. Signal Processing, vol. 42, 1994, pp. 1905-1915.

[10] X. Wang, Z. Wang, and B. O'Dea, "A TOA-Based Location Algorithm Reducing the Errors due to Non-Line-of-Sight (NLOS) Propagation," IEEE Transactions on Vehicular Technology, vol. 52, January 2003, pp. 112-116.

[11] P. C. Chen, "A non-line-of-sight error mitigation algorithm in location estimation," IEEE Wireless Communications Networking Conference, vol. 1, September 1999, pp. 316-320.

[12] C. Y. Lee, "Mobile Communications Engineering," NY, McGraw-Hall, Ch.9, 1993 